\title{
The efficacy of modern spinal orthoses and hip protectors used in the management of osteoporosis: a review
}

\author{
Liora Colobățiu ${ }^{1}$, Simona Mirel ${ }^{1}$
}

1. Department of Medical Devices, Faculty of Pharmacy, Iuliu Hatieganu" University of Medicine and Pharmacy Cluj-Napoca, Romania

\begin{abstract}
Globally, osteoporosis is considered a widespread metabolic bone disease, vertebral fractures being probably one of the most common clinical manifestations encountered in osteoporotic patients. Non-pharmacologic therapies of osteoporosis include, among others, the use of different types of orthoses, such as spinal orthoses or hip protectors.

To date there is a limited number of studies that evaluate the efficacy of spinal orthoses and hip protectors used in osteoporotic patients, therefore providing insufficient information to clinicians regarding these aspects.

This article reviewed the use and efficacy of the most commonly prescribed spinal orthoses and hip protectors in osteoporotic patients. According to a significant number of studies, modern spinal orthoses proved to be effective in the management of vertebral fractures in osteoporotic patients. However, the efficacy of hip protectors in reducing the impact of falls onto the hip and preventing hip fractures still seems to be controversial.

Osteoporosis is a condition that should be treated using a comprehensive approach that includes both pharmacological and non-pharmacological treatment options. Orthoses, such as modern spinal orthoses or back braces and hip protectors might represent an efficacious nonpharmacological option in the management of osteoporotic vertebral or hip fractures.
\end{abstract}

Key words: osteoporosis, vertebral fractures, orthoses, spinal orthoses, hip protectors, efficacy 


\section{INTRODUCTION}

Osteoporosis is a widespread chronic metabolic disease, in the course of which the bone mass continously decreases, usually occurring with increasing age, that predominantly affects postmenopausal women and older people (1). The disease usually leads to an increased risk of bone fractures, especially in the wrist, spine or hips, causing significant disability, morbidity, mortality and expenses $(1,2)$.

The most common clinical consequences of osteoporosis are back pain, hyperkyphosis, limitations of physical functioning and activities of daily living, as well as reduced quality of live (3).

The pharmacological management of osteoporosis includes the administration of specific anti-osteoporotic medication (such as biphosphonates, parathyroid hormone, raloxifen or estrogen), as well as the administration of adequate calcium or vitamin $\mathrm{D}$ supplements and it is mainly aimed at fracture prevention. However, there is a significant number of patients that cannot, or will not, comply with medication regimens (4), mainly due to adverse effects and sometimes even due to therapy costs.

Besides drug prescription, nonpharmacological osteoporosis management it is considered to be an important and very broad concept, as well as a part of the long-term prevention of fractures for men and women, not only for postmenopausal women, but also from childhood to adolescence, pre- and perimenopause (2). Such non-pharmacologic therapies include orthoses, physical exercise programs, lifestyle changes, fall prevention, vertebroplasty or kyphoplasty and usually complement the traditional pharmacological treatment of osteoporosis $(1,4)$.

Modern spinal orthoses, such as toracolumbar braces are often prescribed in the treatment of patients after vertebral fractures caused by osteoporosis in order to increase trunk muscle strenght, reduce pain and improve posture.

Hip fractures are generally considered one of the most dramatic complications of osteoporosis, therefore, reducing the impact of falls onto the hip with hip protectors might represent an effective strategy in preventing such fractures, particularly in nursing home residents (2).

To date there is a limited number of studies that evaluate the efficacy of spinal orthoses and hip protectors used in osteoporotic patients, therefore providing insufficient information to clinicians regarding these aspects.

This article reviews the use and efficacy of the most commonly prescribed spinal orthoses and hip protectors in osteoporotic patients.

\section{Methods}

A systematic literature search from 1990 to 2015 was conducted in PubMed, PubMedCentral and Science Direct. Keywords used to perform the search concerning the most commonly prescribed orthoses and hip protectors in the management of osteoporotic vertebral fractures were "osteoporosis", "orthoses", "non-pharmacological", "spinal" and "hip protectors".

\section{Spinal orthoses}

Orthoses, such as thoracolumbar braces, are often prescribed for osteoporotic patients especially after vertebral compression fractures, and only in a limited number of cases for prophylaxis.

Nowadays, a lot of attention has been payed to the use of flexible spinal orthoses such as Spinomed, Spinomed active men/women or Osteo-med, mainly because some patients find the previously traditionally used rigid braces to be highly constricting and therefore, opt for 
other types of spinal orthoses, such as the modern flexible ones (fig 1).

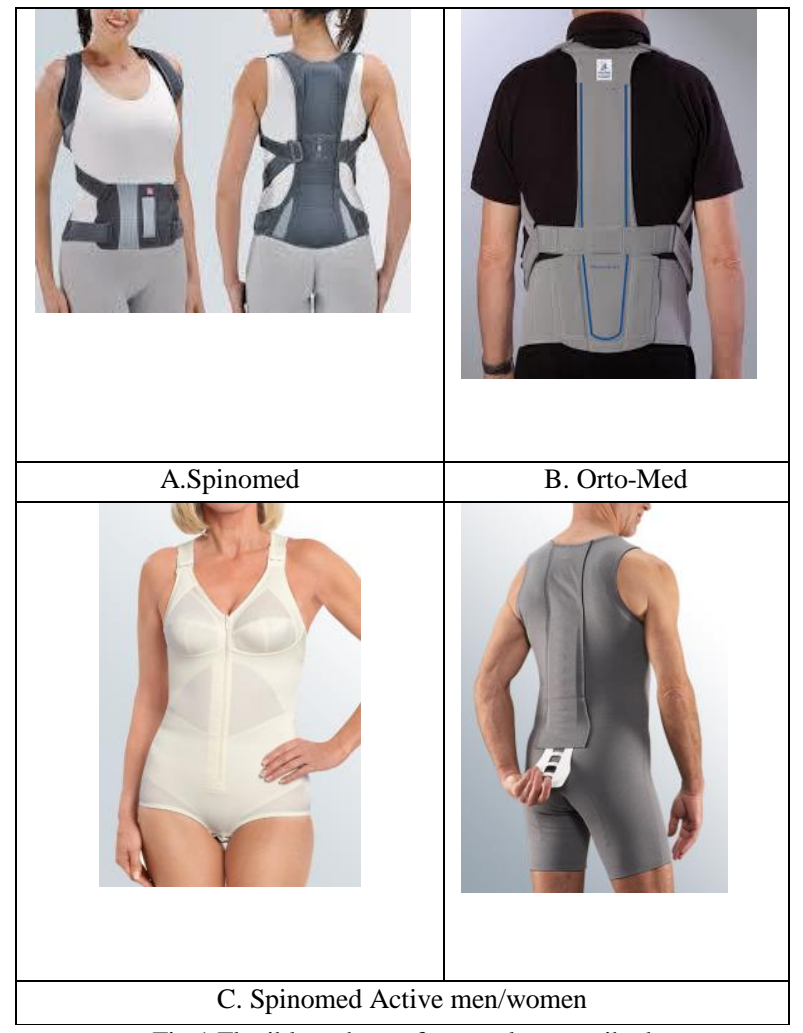

Fig.1 Flexible orthoses frequently prescribed

for osteoporotic patients

Spinomed is a spinal orthosis (back brace) indicated in the treatment of osteoporotic bone collapse in the thoracic and lumbar spine, as well as in the treatment of hyperkyphosis with chronic back pain. It consists of a rigid moldable support contained in a garment that is worn like a backpack and containing weights. The patient friendly "backpack" design permits easy donning and doffing and allows for freedom of shoulder and arm movements. In addition, it does not obstruct thoracic and abdominal breathing and it can be adjusted individually to spinal curvature and body dimensions (5). Rather than forcibly pulling the patient back, the goal of this brace is to serve as a proprioreceptive reminder to the patient to extend their thoracic spine (4).

Osteo-med, similar to Spinomed, is a back brace used in the management of osteoporotic vertebral fractures, and also in avoidance of subsequent damages to spines that can still straighten and when symptoms have already become evident (6).

Certain randomized controlled trials have examined the effects of these spinal orthoses in the management of osteoporosis and especially for the management of osteoporotic vertebral fractures. For instance, a recent study has evaluated the effects of Spinomed on back extensor strenght, back pain and physical functioning in women with osteoporotic vertebral fractures (7). The use of this active spinal orthosis during a 3-month period was associated with an increase in back extensor strenght of $50 \%$. The study also demonstrated a $33 \%$ reduction in back pain and a 6.5 improvement in physical functioning (7).

Another study has examined the efficacy of Spinomed during a larger period of time (6 months). Measurements performed included trunk muscle strenght, angle of kyphosis, body weight, body sway as well as parameters of quality of life such as pain, wellbeing and limitations of daily living. Wearing the orthosis during a 6-months period was associated with a $72 \%$ increase in back extensor strenght, a $44 \%$ increase in abdominal flexor strenght, an $11 \%$ decrease in the angle of kyphosis, 23\% decrease in body sway, a 19\% increase in vital capacity, a $41 \%$ decrease in average pain, an $18 \%$ increase in well-being and a $49 \%$ decrease in limitations of daily living (8).

A comparison study on the efficacy of Spinomed and a soft lumbar orthosis for osteoporotic vertebral fracture showed a significant reduction in the patients' level of pain and limitations of daily life in the case of both types of orthosis, but Spinomed could not provide additional treatment benefits to patients with osteoporotic vertebral fracture regarding pain relief and functional independence improvement at the subacute stage. The authors also concluded that the effects of this medical device in muscle-strenghtening and thoracic kyphotic angle reduction for patients with 
osteoporotic vertebral fracture need to be further verified in a more intensive and longerterm training program (9).

Postural correction in osteoporosis by Osteo-med spinal orthosis has been evaluated in a randomized, placebo-controlled trial. The study included forty women aged $(65.9+/-8.4$ years) with a proven osteoporosis. Some of them were treated with Osteo-med (Thamert) with paravertebral/lumbosacral air chaimber pads, some with the same orthosis without air chamber and the rest with placebo body stocking. The results showed that the orthosis with air chamber pads caused a clinically meaningful trunk support in patients with osteoporotic posture changes (10). Because of the fact that the device does not contain rigid stabilizing elements, the authors concluded that the change in posture could have been a result of muscle activation due to sensomotor stimulation by the air chamber pads (10).

Another study assesed the effects of Osteo-med on gait and pain-induced limitations of activities of daily living in 69 postmenopausal osteoporotic women with and without vertebral fractures. The study demonstrated that wearing such a spinal orthosis introduced a reduction in double support time associated with a beneficial impact on gait stability. Furthermore, there was a positive effect on pain restrictions of activities of daily living, reductions in pain, improvements in back extensor strenght and correction of posture (3).

A study investigating the effect of longterm use and the compliance of spinal orthoses, namely Spinomed, Osteo-med, Spinomed active and Spine-X in postmenopausal women with vertebral fractures, reported that only Spinomed decreased pain and increased trunk muscle strenght after 6 months wearing for at least $2 \mathrm{~h} /$ day. The compliance of wearing an orthosis for 6 months was $66 \%$ (11).

\section{Hip protectors}

Most hip fractures are a result of a direct fall onto the hip. The estimated lifetime risk of hip fracture is about $14 \%$ in postmenopausal women and around $6 \%$ in men, the incidence increasing exponentially with age (12). External hip protectors are normally composed of undergarments (polyprophylene or polyethylene) with padding over the trochanters (4) (fig 2 A). They are designed to absorb the energy from a fall and especially to shunt the energy to the soft tissues around the hip and keep the force on the trochanter below the fracture threshold (2).

The force-attenuation properties of different hip protectors have been demonstrated in several in vitro biochemical studies (13).

One of the most commonly used hip protectors are Safehip (Tytex A/S, Ikast, Denmark) and the KPH hip protector (HRA Pharma, Paris, France) (fig 2 B, C) $(14,15)$.

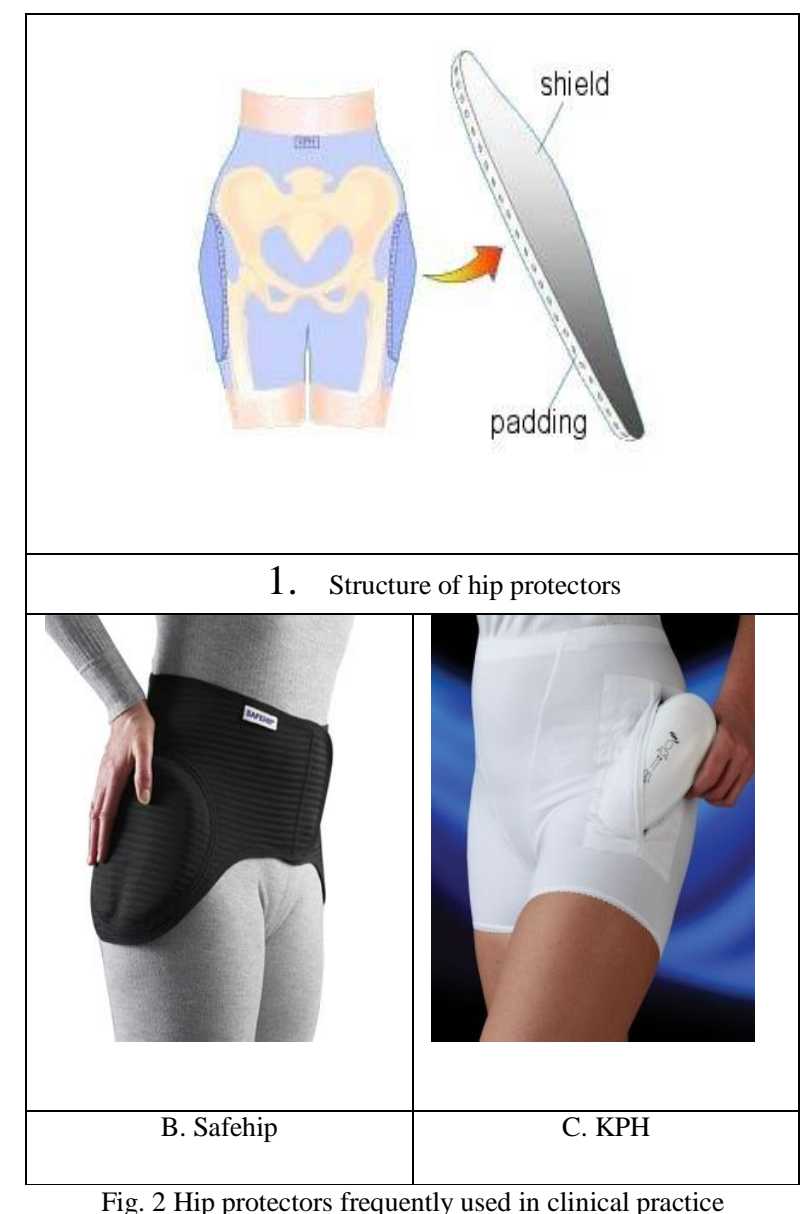


There are different controlled trials that have evaluated the effect of external hip protectors on the incidence of hip fractures, but, unfortunately, the results have been somewhat contradictory.

In a number of studies, hip protectors did significantly reduce the incidence of hip fractures (16-18).

However, a recent study has searched Cochrane Bone, Joint and Muscle Trauma Group Specialised Register (January 2010), The Cochrane Library 2010, Issue 2, MEDLINE (1950 to November 2009), MEDLINE inprocess (30 December 2009), EMBASE (1988 to 2009 week 52), CINAHL (1982 to February 2009), BioMed Central (January 2010) and reference lists of relevant articles in order to evaluate if external hip protectors reduce the incidence of hip fractures in older people following a fall. Analyzing available data, the authors concluded that the effectiveness of the provision of hip protectors in reducing the incidence of hip fracture in older people is still not clearly established, although they may reduce the rate of hip fractures if made available to frail older people in nursing care (19).

One of the main concerns with external hip protectors is poor compliance or even noncompliance, this being considered one of the main limitating factors in the effectiveness of these devices, particular with regard to longterm adherence $(2,4)$ and has also been a key factor contributing to the continuing uncertainty regarding the use of such hip protectors. Factors associated with noncompliance include disconfort on wearing, patients' dislike of their personal appearance with the hip protectors on, as well as disagreement about their fracture risk (20).

\section{Conclusion}

Modern spinal orthoses proved to be effective in the management of vertebral fractures caused by osteoporosis. The use of such orthoses seems to increase muscle strenght (back extensor strenght), reduce pain, improve posture, decrease limitations of daily living and generally contribute to the achievement of a better quality of life for patients suffering from osteoporosis.

Hip protectors are designed in order to reduce the impact of falls onto the hip and to prevent hip fracture. However, randomized controlled trials performed up to date have led to conflicting results, one of the main concerns with hip protectors being poor compliance. 


\section{References}

1. Dionyssiotis Y, Skarantavos

Papagelopoulos P. Modern rehabilitation in osteoporosis, falls, and fractures. Clin Med Insights Arthritis Musculoskelet Disord. 2014;7:33-40.

2. Body J-J, Bergmann P, Boonen S, Boutsen $\mathrm{Y}$, Bruyere O, Devogelaer J-P, et al. Nonpharmacological management of osteoporosis: a consensus of the Belgian Bone Club. Osteoporos Int. 2011;22(11):2769-2788.

3. Schmidt $\mathrm{K}$, Hübscher $\mathrm{M}$, Vogt $\mathrm{L}$, Klinkmüller U, Hildebrandt HD, Fink M, et al. Influence of spinal orthosis on gait and physical functioning in women with postmenopausal osteoporosis. Der Orthopäde. 2012;41(3):200205.

4. Lin JT, Lane JM. Nonpharmacologic management of osteoporosis to minimize fracture risk. Nat Clin Pract Rheumatol. 2008;4(1):20-25.

5. http://mediusa.com/portfolio-item/ spinomed-iv-ap/.

6. http://e-medicalbroker.com/product-eng3664--Thamert-Osteo-Med-Akut.html.

7. Valentin GH, Pedersen LN, Maribo $\mathrm{T}$. Wearing an active spinal orthosis improves back extensor strength in women with osteoporotic vertebral fractures. Prosthet Orthot Int. 2014;38(3):232-238.

8. Pfeifer M, Kohlwey L, Begerow B, Minne HW. Effects of two newly developed spinal orthoses on trunk muscle strength, posture, and quality-of-life in women with postmenopausal osteoporosis: a randomized trial. Am J Phys Med Rehabil.2011;90(10):805-815

9. Li M, Law S, Cheng J, Kee H, Wong MS. A comparison study on the efficacy of SpinoMed® and soft lumbar orthosis for osteoporotic vertebral fracture. Prosthet Orthot Int. 2015;39(4):270-276.

10. Vogt L, Hübscher M, Brettmann K, Banzer W, Fink M. Postural correction by osteoporosis orthosis (Osteo-med): a randomized, placebo- controlled trial. Prosthet Orthot Int. 2008;32(1):103-110.

, 11. Dionyssiotis Y, Trovas G, Thoma S, Lyritis G, Papaioannou N. Prospective study of spinal orthoses in women. Prosthet Orthot Int. 2015;39(6):487-495.

12. Lauritzen JB. Hip fractures: incidence, risk factors, energy absorption, and prevention. Bone. 1996;18(1 Suppl):65S - 75S.

13. Kannus P, Parkkari J, Poutala J. Comparison of force attenuation properties of four different hip protectors under simulated falling conditions in the elderly: an in vitro biomechanical study. Bone. 1999;25(2):229235.

14.

http://tytex.com/products/hipprotection/safehip-airx/

15. http://www.kphprotector.com/ product.html 16. Lauritzen JB, Petersen MM, Lund B. Effect of external hip protectors on hip fractures. Lancet (London, England). 1993;341(8836):11-13.

17. Kannus P, Parkkari J, Niemi S, Pasanen M, Palvanen M, Järvinen M, et al. Prevention of hip fracture in elderly people with use of a hip protector. N Engl J Med. 2000;343(21):15061513.

18. Harada A, Mizuno M, Takemura M, Tokuda H, Okuizumi H, Niino N. Hip fracture prevention trial using hip protectors in Japanese nursing homes. Osteoporos Int. 2001;12(3):215-221.

19. Gillespie WJ, Gillespie LD, Parker MJ. Hip protectors for preventing hip fractures in older people. Cochrane database Syst Rev. 2010;(10):CD001255.

20. Patel S, Ogunremi L, Chinappen U. Acceptability and compliance with hip protectors in community-dwelling women at high risk of hip fracture. Rheumatology. 2003;42(6):769-772 